\title{
CIOs müssen sich auf Sicherheit und Innovation konzentrieren
}

Rudolf Kergaßner

\begin{abstract}
Angesichts der aktuellen Diskussion werden Unternehmen verstärkt in die IT-Sicherheit investieren. Damit die IT dann überhaupt noch Innovationen voranbringen kann, ist die Automatisierung des laufenden Betriebs notwendiger denn je. Die Rolle des $\mathrm{CIO}$ muss dementsprechend neu definiert werden.
\end{abstract}

Die Arbeit des Chief Information Officer (CIO) wurde in den letzten Monaten nicht einfacher. Die IT ist heute ja nicht nur mit den herkömmlichen kriminellen Angriffen, sondern wie neuerdings immer wieder zu erfahren ist - auch mit diversen Ausspähaktionen auf höchstem Niveau konfrontiert. Der CIO ist für die Sicherheit der IT und damit für die Integrität eines Unternehmens (mit-)verantwortlich - zugleich soll er aber auch die Innovationen voranbringen, die zur Aufrechterhaltung der Wettbewerbsfähigkeit notwendig sind.

Tatsächlich beansprucht die Aufrechterhaltung des Betriebs der vorhandenen Systeme bereits 70 bis 80 Prozent der ITBudgets. Da viele Unternehmen vor dem Hintergrund der aktuellen Debatte in die IT-Sicherheit investieren wollen und müssen, ist absehbar, dass für echte Innovationen in Zukunft tendenziell noch weniger Budget bereitstehen wird. Wichtige Themen wie Big Data oder Cloud-Computing werden damit nicht oder nur unzureichend besetzt werden können.

In dieser Situation ist auch eine Neuausrichtung der Rolle des CIO notwendig: Er muss seine Aufgabe künftig vor allem in der Gewährleistung eines hohen Sicherheitsniveaus sowie in der Bereitstellung von Ressourcen für die Erhöhung der Produktivität der Geschäftsbereiche sehen. Die bloße Aufrechterhaltung des Betriebs vorhandener Systeme darf dagegen nicht länger im Zentrum stehen.

Der alltägliche IT-Betrieb muss heute hoch-automatisiert funktionieren. Es gibt längst entsprechende Lösungen, die auch sehr anspruchsvolle Aufgaben übernehmen können. Unternehmen können damit die Effizienz ihres IT-Betriebs erheblich steigern und so die Handlungsfreiheit gewinnen, die sie für die Herausforderungen bei den Themen Sicherheit und Innovation dringend benötigen - und die CIOs können sich auf diese strategischen Aufgaben konzentrieren.

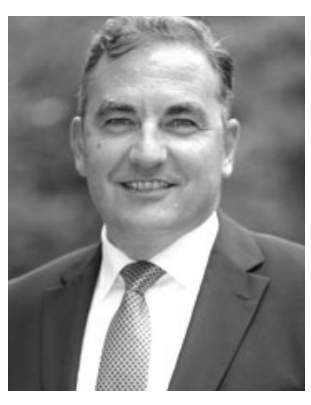

Rudolf Kergaßner ist Managing Director von IPsoft Deutschland in Frankfurt. 